\title{
Accurate band mapping via photoemission from thin films
}

\author{
A. Mugarza, ${ }^{1,2}$ A. Marini, ${ }^{2}$ T. Strasser, ${ }^{3}$ W. Schattke, ${ }^{3}$ A. Rubio, ${ }^{2,4}$ F. J. García de Abajo, ${ }^{2}$ J. Lobo, ${ }^{5}$ E. G. Michel, ${ }^{5}$ \\ J. Kuntze, ${ }^{2}$ and J. E. Ortega ${ }^{1,2}$ \\ ${ }^{1}$ Departamento de Física Aplicada I, Universidad del País Vasco, Plaza Oñate 2, E-20018 San Sebastián, Spain \\ ${ }^{2}$ Centro Mixto CSIC/UPV and DIPC, 20018 San Sebastián, Spain \\ ${ }^{3}$ Institut für Theoretische Physik und Astrophysik, Christian-Albrechts-Universität zu Kiel, Leibnizstrasse 15, D-24098 Kiel, Germany \\ ${ }^{4}$ Departamento de Física de Materiales, Universidad del País Vasco, E-20018 San Sebastián, Spain \\ ${ }^{5}$ Departamento de Física de la Materia Condensada and Instituto Nicolás Cabrera, U. Autónoma de Madrid, 28049 Madrid, Spain
}

(Received 23 July 2003; published 22 March 2004)

\begin{abstract}
Electron bands in solids can be determined in angle-resolved photoemission experiments from thin films, where the perpendicular wave vector $\left(k_{\perp}\right)$ uncertainty that characterizes photoemission from bulk crystals is removed. However, the comparison with state-of-the-art quasiparticle band-structure calculations has never been done. In this work we have mapped both initial-state (occupied) and final-state (empty) $E\left(k_{\perp}\right)$ bands along the $\Lambda$ axis of aluminum, from photon-energy- and thickness-dependent quantum-well spectra of aluminum films. For final states the best fit is obtained with inverse low-energy electron diffraction band structure calculations. For initial-state bands of $\mathrm{Cu}$ and $\mathrm{Al}$, thin-film data display excellent agreement with bulk quasiparticle theory, suggesting the use of thin films as model systems to investigate fine effects in the crystal band structure.
\end{abstract}

DOI: 10.1103/PhysRevB.69.115422

PACS number(s): 73.20.-r, 79.60.Bm

An appropriate description of the electron band structures is of general interest as a fundamental property of crystalline solids that explains most of their observable behavior. Many different spectroscopic techniques probe electronic states, but only photoemission from well-defined crystal surfaces allows the thorough determination of $E(\mathbf{k})$ band dispersions. ${ }^{1}$ Angle-resolved, valence-band photoemission spectra are generally dominated by peaks that correspond to the so-called vertical transitions from initial to final bulk states, i.e., those where the wave vector $\mathbf{k}$ is conserved in the reduced Brillouin zone. The energy and the wave vector parallel to the surface can be determined with a high accuracy depending on the system resolution. However, the broken symmetry at the surface gives rise to a fundamental uncertainty that affects the perpendicular component of the wave vector $k_{\perp}$. Occupied bands are usually mapped assuming free-electron-like final-states to define $k_{\perp}$, but the actual final state band structure is often more complex as proved in constant initial-state (CIS) experiments. Such final states can be determined experimentally by very low-energy electron diffraction(VLEED). ${ }^{2,3}$ However, this method is limited to high-enough reflectivity, i.e., electron energies below $30 \mathrm{eV}$.

The $k_{\perp}$ uncertainty is removed in thin films, where $k_{\perp}$ is fixed by thickness and boundary conditions at the surface interface. ${ }^{4}$ This leads to discrete quantum-well (QW) states in the photoemission spectra, with peaks at $k_{\lrcorner}(E)$ values that fulfill constructive interference conditions. ${ }^{5-10}$ If the film is not too thin and the crystal structure is the same as in bulk materials, $k_{\perp}(E)$ values actually sample bulk $E(\mathbf{k})$ bands at discrete $k_{\perp}$ points. ${ }^{4}$ Furthermore, if it is assumed that boundary conditions at the surface and the interface do not change by varying the film thickness, $k_{\perp}(E)$ can be obtained directly from the so-called QW structure plot, i.e., the QW peak energy distribution as a function of film thickness. ${ }^{5-10}$ Thus, the fundamental $k_{\perp}$ uncertainty in bulk crystal photoemission is transferred to an experimentally, controllable thickness uncertainty in thin films.
In this paper we compare initial- and final-state bands measured in thin $\mathrm{Al}$ and $\mathrm{Cu}$ films with state-of-the-art band calculations. Although thin films have been already used to map initial-state bands in a number of systems, ${ }^{5-8}$ the comparison with quasiparticle band theory is still missing. Especially in noble metals, where disagreement is found with ground-state bands. ${ }^{6,9}$ Here, we show that experiments and quasiparticle theory agree for $\mathrm{Al}$ and $\mathrm{Cu}$ films with unprecedented accuracy, demonstrating the validity of the thin-film approach. Such accuracy opens up a new way to explore correlation or electron-phonon coupling effects in the band structure near $E_{F}$. Furthermore, detailed knowledge of the initial state allows us to determine final states by photonenergy-dependent photoemission in the corresponding bulk crystal. ${ }^{9}$ We show that two different experiments are not strictly necessary, since the final state can be directly mapped using the same film with CIS-like photoemission, i.e., by measuring photon-energy-dependent quantum-well intensity. This allows mapping several photoemission final-state bands beyond $30 \mathrm{eV}$, and comparing data with different theoretical approaches. We find that complex, final-state band-structure calculations provide the best fit.

Photoemission data were acquired with a VSW angleresolved analyzer coupled to the undulator VUV beam line at the Synchrotron ELETTRA in Trieste (Italy). The light was $p$ polarized with an incidence angle of $70^{\circ}$ from surface normal. The energy resolution was $7+40 \mathrm{meV}$ (photons + electrons). $\mathrm{Si}(111)$ wafers were prepared by repeatedly flashing to $1500 \mathrm{~K}$. Al films were evaporated with a deposition rate of $\sim 0.5 \AA / \mathrm{min}$ from a BN crucible operating at a pressure below $2 \times 10^{-10}$ mbar, which ensures clean Al films. The thickness is determined with a quartz microbalance, and cross checked by comparing quantum-well spectra with those of Aballe et al. ${ }^{10}$ In order to obtain sharp $\mathrm{Al} / \mathrm{Si}$ interfaces and very smooth films we follow the procedure given in Ref. 10, i.e., depositing a $2 \mathrm{ML} \mathrm{Al}$ buffer film at $120 \mathrm{~K}$ with a short 
(a)

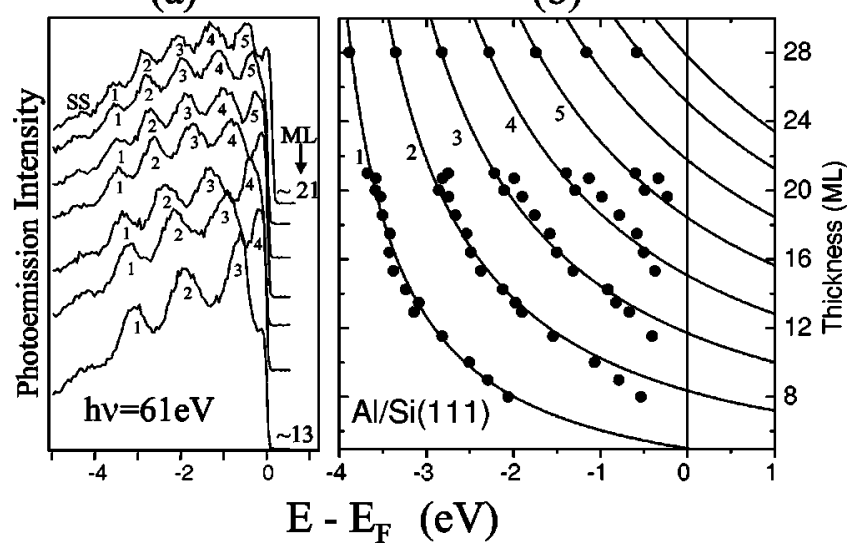

FIG. 1. (a) Valence-band spectra from thin $\mathrm{Al}(111)$ films grown on Si. QW state peaks are clearly observed. Their energies at a given thickness are correspondingly represented in the structure plot of (b). The lines are fit to data points using a phase accumulation model that determines the band dispersion $E\left(k_{\perp}\right)$ for bulk Al.

postannealing to $500 \mathrm{~K}$. Subsequent layers in thicknessdependent measurements were deposited on top of this buffer layer at $300 \mathrm{~K}$, which was the measuring temperature. The quality of the film is inherently proved by the sharpness of the quantum-well peaks in the spectra. Low-energy electron diffraction(LEED) analysis and surface x-ray diffraction experiments indicate that $\mathrm{Al}$ films exhibit the same crystal structure as bulk Al. ${ }^{11}$

Figure 1(a) shows a typical series of photoemission spectra for varying $\mathrm{Al}$ thickness taken at $h \nu=61 \mathrm{eV}$. As we increase the number of layers, new quantum-well peaks cross the Fermi level, shifting towards the band edge at around $-4.6 \mathrm{eV} .{ }^{12}$ The very last peak at this energy corresponds to the $\mathrm{Al}(111)$ surface state. Figure 1(b) contains the structure plot with the quantum-well peak energy dispersion as a function of thickness. It includes data from Fig. 1(a) and another series of spectra from $8 \mathrm{ML}$ to $13 \mathrm{ML}$. Most of the data points lie below the absolute substrate band gap at $\sim-0.5 \mathrm{eV}$, and hence they are resonances rather than pure quantum-well states, similar to those found in other systems like $\mathrm{Cu}$ films. ${ }^{6}$ The thin lines fit simultaneously all data points using the expression

$$
d_{n}(E)=\frac{n-1+\phi(E)}{1-k_{\perp}(E)},
$$

where $d_{n}(E)$ is the film thickness in layers, $k_{\perp}(E)$, in units of the fundamental wave vector $2 \pi / a$, is a parametrized band dispersion obtained from the two-band model, and $\phi(E)$ accounts for the total phase shift at the surface and the interface. The fit is very good, except for the small change of slope near $E_{F}$, which is probably due to the presence of the edge of the band gap in the Si substrate. ${ }^{10,13}$ Equation (1) can be deduced from both the envelope function model of the wave function inside a quantum well, ${ }^{6}$ or the phase accumulation model. ${ }^{14,5}$ The phase shift can be either assumed to be a linear function of the energy with two fitting parameters, ${ }^{6}$ or approached with a more realistic WKB function for the
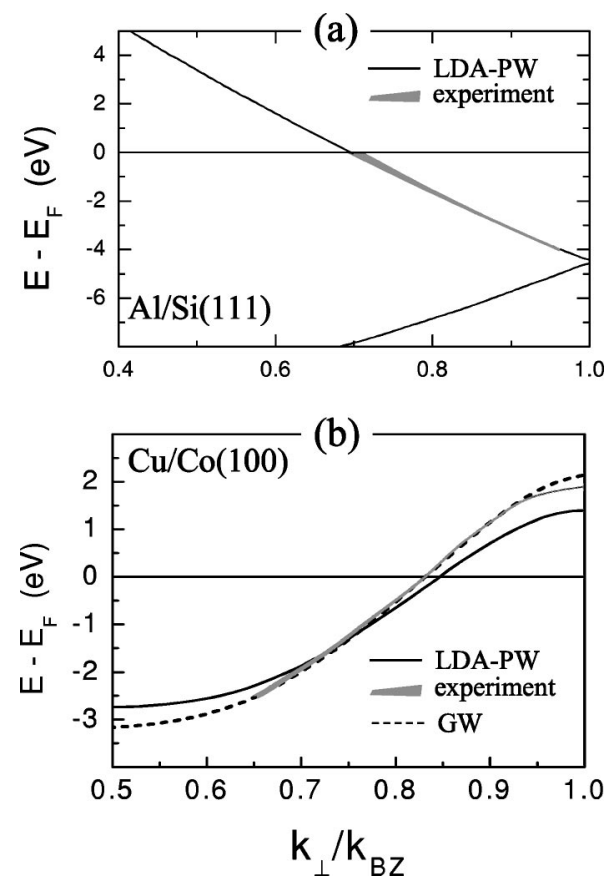

FIG. 2. (a) Band dispersion for $\mathrm{Al}$ along the $\Lambda$ axis as determined in the structure plot analysis of Fig. 2(b) (thick gray line). The line thickness accounts for the experimental error. The resulting band fits sharply to a PW-LDA band calculation for bulk Al. (b) Band dispersion along the $\Delta$ axis measured for $\mathrm{Cu}(100)$ films. Direct and inverse photoemission data have been taken from Refs. 6 and 8 . In this case, the fit to GW bands is excellent.

vacuum barrier and still a linear function for the interface. ${ }^{10}$ The fact is that the fit is strongly dependent on $k_{\perp}(E)$, which determines the density of lines (QW periodicity), and rather insensitive to $\phi(E)$. Thus, $k_{\perp}(E)$ is obtained with a high accuracy that is basically limited by the thickness uncertainty.

The experimental $k_{\perp}(E)$ obtained from the fit to the structure plot in Fig. 1(b) is shown in Fig. 2(a) using a thick gray line. The line thickness accounts for the experimental error, which is maximum at the Fermi energy $\Delta k_{\perp, \max }$ $=0.006 \AA^{-1}$. In Fig. 2(b) we also show the band dispersion determined from a similar structure plot analysis of $\mathrm{Cu}(100)$ quantum well states in thin films grown on fcc $\mathrm{Co}(100){ }^{6,8}$ Thin solid lines in Fig. 2 represent first-principle densityfunctional bands calculated in the local-density approximation (LDA) along the $\Lambda$ and the $\Delta$ axis for bulk $\mathrm{Al}$ and $\mathrm{Cu}$, respectively. Thin dotted lines contain self-energy corrections, calculated within the standard GW approximation, ${ }^{15}$ which are considerable in $\mathrm{Cu}$ and negligible in Al. Except at the $X_{4}^{\prime}$ edge in $\mathrm{Cu}$, which is obtained from the asymptotic limit of the $d_{n}(E)$ lines, quasiparticle bands and data agree extremely well. This demonstrates the appropriateness of both the experimental and the theoretical approaches. The self-energy correction to ground-state bands in $\mathrm{Cu}$ can explain the deviations observed between experiment and theory in $\mathrm{Ag}(100)$ films. ${ }^{9}$ Such correction is expected from the partial $d$ character of the $s, p$ band in $\mathrm{Cu}$ and $\mathrm{Ag},{ }^{16}$ in contrast to the pure $s, p$ character in Al. As shown in Fig. 2(b) the measured $s, p$ band is wider than the ground-state band. This 


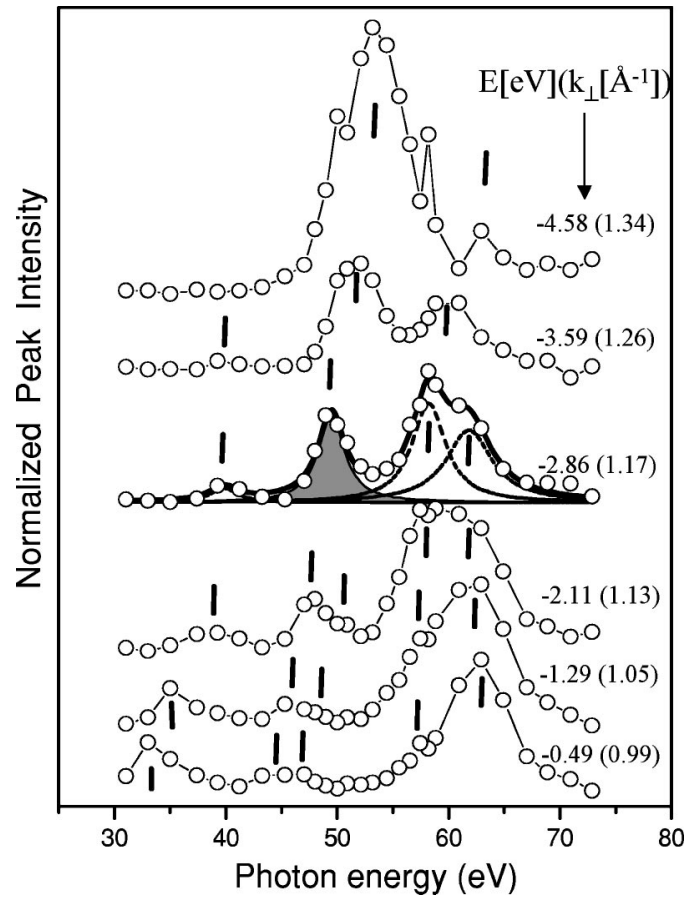

FIG. 3. Normalized, photon-energy-dependent intensity of the QW and surface-state peaks for the $20 \mathrm{ML}$ Al thin film in Fig. 2(a). We observe fine features that correspond to transitions to different final-state bands. Their dispersion is discretely sampled at each curve.

indicates a lower hybridization with $d$ bands, which are indeed shifted down in the excited state. ${ }^{15}$

Assuming the vertical transition scheme in the reduced Brillouin zone, we have an accurate set of $k_{\perp}(E)$ values for each QW peak of Fig. 1(a). This allows one to probe the photoemission final state in the crystal by measuring the QW photon-energy-dependent intensity. ${ }^{8}$ This is done in Fig. 3 for the $20 \mathrm{ML}$ spectrum of Fig. 1(a). Each peak in Fig. 3 corresponds to a direct transition to a final state. Note that the energy range is unreachable by VLEED. ${ }^{3}$ Distinct peaks (marked with ticks) can be consistently fitted using Lorentzian lines. As a case example, we include the results of the fit for one of the curves. The full width at half maximum for different features in all curves is always lower than $\Delta E$ $=6 \mathrm{eV}$. Broader features could hide more than one transition, since they can be as sharp as $\Delta E=3 \mathrm{eV}$, like the shaded peak at $h \nu=49 \mathrm{eV}\left(E-E_{F}=47 \mathrm{eV}\right.$, when correcting for the QW binding energy). At around $50 \mathrm{eV}$, the $\mathrm{QW}$ peak broadening $\left(\Delta k_{\perp}\right)$ contains the contributions of both the finite photoelectron escape depth $\left(\Delta k_{f}\right)$ and the initialstate broadening $\left(\Delta k_{i}\right)$, which is due to the finite thickness of the film. ${ }^{8}$ For a numerical estimate we assume $\Delta E$ $=3 \mathrm{eV}, E=47 \mathrm{eV}$, and the group velocity as deduced from our band mapping in Fig. $4\left(\partial E / \partial k_{\perp}\right)^{-1}=(18 \mathrm{eV} \AA)^{-1}$. We obtain $\Delta k_{\perp}=\Delta E\left(\partial E / \partial k_{\perp}\right)^{-1}=0.167 \AA^{-1}$. The thickness broadening can be estimated from the uncertainty principle to be of the order of $\Delta k_{i} \sim k_{Z B} / 20=0.067 \AA^{-1} .^{8}$ Therefore the escape depth broadening is $\Delta k_{f} \sim 0.100 \AA^{-1}$, and $\lambda$ $=1 / \Delta k_{f} \sim 10 \AA$. This value is three times larger than the calculated inelastic mean-free path for bulk $\mathrm{Al},{ }^{17}$ although

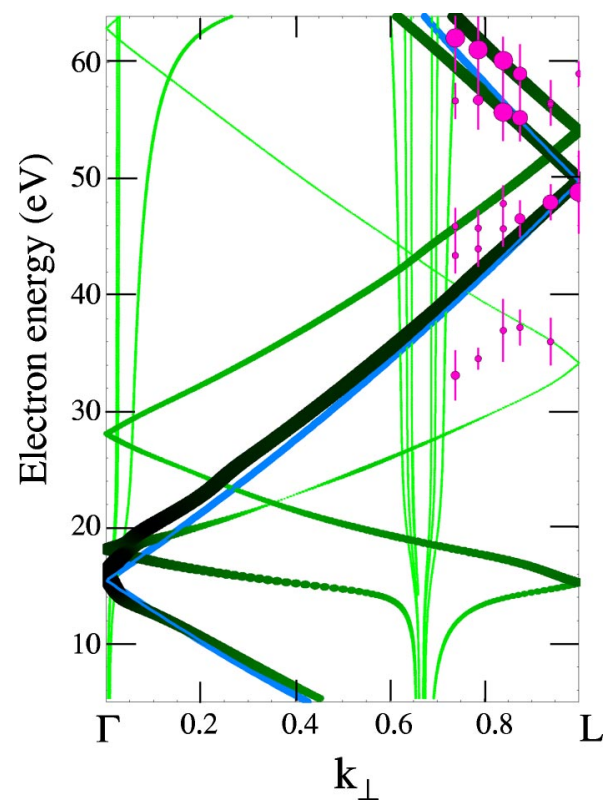

FIG. 4. Final-state band mapping. Data points correspond to the peaks (tick marks) in Fig. 3, with their size reflecting the peak intensity. The blue line is the primary-cone, free-electron band in an inner potential $E_{F}-V_{0}=8.8 \mathrm{eV}$. The green lines are complex bandstructure calculations. Both line intensity and thickness are proportional to the photoemission matrix element calculated with pseudopotential bands for the thin film.

such deviation appears reasonable given the strong directional character of the escape depth. Notice that the width of the surface-state cross section peak at $54 \mathrm{eV}$ is the same as the one measured from a $\mathrm{Al}(111)$ single crystal. ${ }^{12}$ From this width it is also possible to obtain an estimate of the escape depth $\lambda=7 \AA,{ }^{12}$ which is consistent with the present analysis.

The spectra of Fig. 3 sample final-state bands at six different $k_{\perp}$ values. The result is shown in a reduced zone scheme in Fig. 4. The size of the data points represents the relative intensity of the transitions in Fig. 3, and the error bars reflect the peak width. GW self-energy, quasiparticle corrections do not provide a reasonable description of the photoemission final state in this case. In this high-energy excited state, electrons leave the solid before they are dressed by the screening cloud, which is the main physical mechanism described by GW self-energy calculations. For a comparison with the experiment we have separately tested bulk LDA bands and complex band-structure calculations. The latter give the best fit and are included in Fig. 4 as green lines. The solutions of the bulk complex band structure are calculated with an empirical pseudopotential, ${ }^{18}$ including an imaginary part in the optical potential $(1.8 \mathrm{eV}$ at $50 \mathrm{eV}$ kinetic energy) that describes the damping of the wave function towards the crystal. Furthermore, we have determined the final photoemission state by matching these solutions to the vacuum solution. The latter defines an inverse LEED final-state wave function in the presence of a step potential to represent the surface. ${ }^{19}$ Inside the crystal, the final state is composed of a sum over bulk solutions of different $k_{\perp}$ and the magnitude of the corresponding expansion coefficients is 
indicated by the thickness of the green lines in Fig. 4, such that the bands which contribute significantly to photoemission can be identified. ${ }^{20}$ Theory and experiment exhibit general agreement in intensity and the dispersion of the bands are reasonably well probed, but theoretical bands disperse faster away from $k_{Z B}$. Such deviation could be related to a stronger hybridization with $d$-like bands around $40 \mathrm{eV}$, which would not be appropriately addressed within the empirical pseudopotential. By contrast, LDA bands of $\Lambda_{1}$ symmetry display the same group velocity as the more intense, freeelectron-like experimental bands of Fig. 4.

In summary, state-of-the-art GW theory and photoemission experiments using thin films show excellent agreement for occupied valence bands in metals. Furthermore, initialstate bands allows mapping high-energy bands beyond 30 $\mathrm{eV}$, where the reported theory describes correctly the observed strong deviation with respect to free-electron bands. The results suggest the use of thin films as model systems to test small many-body corrections to the band structure near the Fermi energy, such as correlation effects. Also, highresolution, low-temperature photoemission from thin films can be devised as a suitable technique to measure absolute electron-phonon couplings, both from the QW peak width analysis (imaginary part) and from the exact band dispersion (real part).

This work has been supported in part by the Universidad del País Vasco (A.Mu. and J.E.O. under Contract No. 00057.240-EA-13668/2001, and A.Ma., A.R., and F.J.G.A. under Contract No. 00206.215-13639/2001), the European Community (EC) research training network NANOPHASE [A.Ma. and A.R. (Grant No. HPRN-CT-2000-00167)], and the Spanish Ministerio de Ciencia y Tecnología [A.Ma., A.R., and F.J.G.A. (Grant No. MAT2001-0946), and J.L. and E.G.M. (BFM2001-0244)]. Experiments at Elettra were supported by the EC via the Access to Research Infrastructure actions within the Improving Human Potential Program.
${ }^{1}$ F.J. Himpsel, Adv. Phys. 32, 1 (1983).

${ }^{2}$ M. Lindroos, H. Pfnür, and D. Menzel, Phys. Rev. B 33, 6684 (1986)

${ }^{3}$ V.N. Strocov, R. Claessen, G. Nicolay, S. Hufner, A. Kimura, A. Harasawa, S. Shin, A. Kakizaki, P.O. Nilsson, H.I. Starnberg, and P. Blaha, Phys. Rev. Lett. 81, 4943 (1998).

${ }^{4}$ P.D. Loly and J.B. Pendry, J. Phys. C 16, 423 (1983).

${ }^{5}$ S.A. Lindgren and L. Walldén, Phys. Rev. Lett. 59, 3003 (1987); 61, 2894 (1988).

${ }^{6}$ J.E. Ortega, F.J. Himpsel, G.J. Mankey, and R.F. Willis, Phys. Rev. B 47, 1540 (1993).

${ }^{7}$ M.A. Mueller, T. Miller, and T.-C. Chiang, Phys. Rev. B 41, 5214 (1990); D. Claesson, S.-A. Lindgren, and L. Walldén, ibid. 60, 5217 (1999); D.-A. Luh, J.J. Paggel, T. Miller, and T.-C. Chiang, Phys. Rev. Lett. 84, 3410 (2000); D.V. Vyalykh, A.M. Shikin, G.V. Prudnikova, A.Yu. Grigor'ev, A.G. Starodubov, and V.K. Adamchuk, Phys. Solid State 44, 164 (2002); L. Aballe, C. Rogero, and K. Horn, Surf. Sci. 518, 141 (2002).

${ }^{8}$ A. Mugarza, J.E. Ortega, A. Mascaraque, E.G. Michel, K.N. Altmann, and F.J. Himpsel, Surf. Sci. 482-485, 464 (2001).

${ }^{9}$ J.J. Paggel, T. Miller, and T.-C. Chiang, Phys. Rev. B 61,
1804 (2000).

${ }^{10}$ L. Aballe, C. Rogero, S. Gokhale, S. Kulkarni, and K. Horn, Surf. Sci. 482-485, 488 (2001).

${ }^{11}$ A. Mascaraque et al. (unpublished).

${ }^{12}$ S.D. Kevan, N.G. Stoffel, and N.V. Smith, Phys. Rev. B 31, 1788 (1985).

${ }^{13}$ T.G. Pedersen, K. Pedersen, P.K. Cristensen, J. Rafaelsen, N. Skivesen, Z. Li, and S.V. Hoffmann, Surf. Sci. 516, 127 (2002).

${ }^{14}$ P.M. Echenique and J.B. Pendry, J. Phys. C 11, 2065 (1978).

${ }^{15}$ G. Onida, L. Reining, and A. Rubio, Rev. Mod. Phys. 74, 601 (2002), and references therein

${ }^{16}$ A. Marini, G. Onida, and R. Del Sole, Phys. Rev. Lett. 88, 016403 (2001); A. Marini, R. Del Sole, A. Rubio, and G. Onida, Phys. Rev. B 66, 161104 (2002).

${ }^{17}$ S. Tanuma, C.J. Powell, and D.R. Penn, Surf. Interface Anal. 17, 911 (1991).

${ }^{18}$ M. Cohen and T. Bergstresser, Phys. Rev. 141, 789 (1966).

${ }^{19}$ J.B. Pendry, J. Phys. C 2, 2273 (1969); H. Bross, Surf. Sci. 213, 215 (1989).

${ }^{20}$ T. Strasser, F. Starrost, C. Solterbeck, and W. Schattke, Phys. Rev. B 56, 13326 (1997). 